\title{
Strain analysis of a Ge micro disk using precession electron diffraction
}

Cite as: J. Appl. Phys. 126, 235701 (2019); https://doi.org/10.1063/1.5113761

Submitted: 07 June 2019 . Accepted: 23 November 2019 . Published Online: 16 December 2019

Aneeqa Bashir (iD), Ross W. Millar (D), Kevin Gallacher (D), Douglas J. Paul (D), Amith D. Darbal (D), Robert Stroud (D), Andrea Ballabio (D), Jacopo Frigerio (D), Giovanni Isella (D), and lan MacLaren (D)

\section{COLLECTIONS}

This paper was selected as Featured

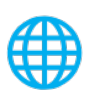

View Online

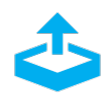

Export Citation

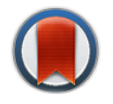

CrossMark

\section{ARTICLES YOU MAY BE INTERESTED IN}

Giant unidirectional spin Hall magnetoresistance in topological insulator - ferromagnetic semiconductor heterostructures

Journal of Applied Physics 126, 233903 (2019); https://doi.org/10.1063/1.5134728

Atomic-scale quantitative analysis of implanted $\mathrm{Mg}$ in annealed $\mathrm{GaN}$ layers on free-standing GaN substrates

Journal of Applied Physics 126, 235704 (2019); https://doi.org/10.1063/1.5132345

Predicted high-temperature superconductivity in cerium hydrides at high pressures

Journal of Applied Physics 126, 235901 (2019); https://doi.org/10.1063/1.5130583

Journal of Applied Physics
SPECIAL TOPIC:

Antiferromagnetic Spintronics 


\title{
Strain analysis of a Ge micro disk using precession electron diffraction
}

\author{
Cite as: J. Appl. Phys. 126, 235701 (2019); doi: 10.1063/1.5113761 \\ Submitted: 7 June 2019 . Accepted: 23 November 2019 . \\ Published Online: 16 December 2019
}

\begin{abstract}
Aneeqa Bashir, ${ }^{1}$ (D) Ross W. Millar, ${ }^{2}$ (D) Kevin Gallacher, ${ }^{2}$ (D) Douglas J. Paul, ${ }^{2}$ (D) Amith D. Darbal, ${ }^{3}$ Robert Stroud, $^{3}$ (D) Andrea Ballabio, ${ }^{4}$ (D) Jacopo Frigerio, ${ }^{4}$ (D) Giovanni Isella, ${ }^{4}$ (D) and Ian MacLaren ${ }^{7, a)}$ (D)

\author{
AFFILIATIONS \\ ${ }^{1}$ School of Physics and Astronomy, University of Glasgow, Kelvin Building, University Avenue, Glasgow G12 8QQ, United Kingdom \\ ${ }^{2}$ School of Engineering, University of Glasgow, Rankine Building, Oakfield Avenue, Glasgow G12 8LT, United Kingdom \\ ${ }^{3}$ NanoMEGAS USA, 1095 W Rio Salado Parkway, Tempe, Arizona 85281, USA \\ ${ }^{4}$ L-NESS, Dipartimento di Fisica del Politecnico di Milano, Polo Territoriale di Como, Via Anzani 42, Como I-22100, Italy
}

a) Author to whom correspondence should be addressed: ian.maclaren@glasgow.ac.uk

\begin{abstract}
The recently developed precession electron diffraction (PED) technique in scanning transmission electron microscopy has been used to elucidate the local strain distribution and crystalline misorientation in a CMOS fabricated strained Ge microdisk structure grown on a Si substrate. Tensile strained Ge and GeSn structures are considered to be potential CMOS compatible optical sources, as both Sn alloying and strain can lead to a direct band-structure and lasing. The ability to take nanometer resolution, experimental measurements of the cross-sectional strain distribution, is important to understand modal gain and, therefore, ultimate device performance. In this work, we demonstrate PED techniques to measure the cross-sectional strain field in tensile Ge microdisks strained by SiN stressors. The strain maps are interpreted and compared with a finite element model of the strain in the investigated structure, which shows good agreement, and, therefore, highlights the applicability of PED techniques for mapping strained photonic structures. The technique also allows for the observation of strain relaxation due to dislocation pileup, further demonstrating the benefit of such experimental techniques.
\end{abstract}

\section{Published under license by AIP Publishing. https://doi.org/10.1063/1.5113761}

\section{INTRODUCTION}

It is only recently that $\mathrm{Ge}$ has made ground in the standard $\mathrm{Si}$ technology for being compatible with CMOS processing. ${ }^{1} \mathrm{Ge}$ on $\mathrm{Si}$ has been established as a low cost photonic component with promising applications including photodetectors ${ }^{2}$ and modulators. ${ }^{3,4}$ In the last decade, Ge has also been investigated for active optical sources and lasers. This has been driven by the fact that, despite being an indirect bandgap material, the direct $\Gamma$-valley is only $140 \mathrm{meV}$ above the L-valley, meaning that electrical population of the direct valley is possible. Lasing has been demonstrated in Ge Fabry-Pérot cavities with high degenerate n-type doping and low tensile strain from growth on $\mathrm{Si}^{5-7}$ This doping reduces the required injection to populate the $\Gamma$, thereby minimizing the excess injected hole population, and the associated strong intervalence and free carrier absorption (FCA). Degenerate phosphorous doping, however, leads to a reduced excess carrier recombination lifetime ${ }^{8}$ and still leads to free carrier losses, which produces high lasing thresholds and has limited the number of demonstrations of room temperature $\mathrm{Ge} / \mathrm{GeSn}$ lasing with low strain.

Applying biaxial tensile strain to $\mathrm{Ge}$ causes the $\Gamma$-valley to lower the energy at a greater rate than the L-valley, leading to a crossover to a direct band-structure at $\sim 1.7 \%$. ${ }^{9}$ This reduces the required injection to populate the $\Gamma$-valley, and, therefore, reduces FCA/intervalence band losses. Two major approaches have been used for strain engineering Ge. Locally undercut photonic wires or microbridges can locally amplify the low tensile strain from the epitaxial growth of $\mathrm{Ge}$ on $\mathrm{Si}^{10}{ }^{10}$ resulting in large uniaxial strains of up to $5.7 \%{ }^{11}$ at room temperature. Lasing has been reported at low temperatures by this approach. ${ }^{12} \mathrm{SiN}$ stressors have also been used to externally apply strain to compact microdisk structures, with sufficient biaxial tensile strain to transition to a direct bandgap band structure. ${ }^{9,13}$ Recently, lasing at low temperatures has been demonstrated in highly tensile strained Ge microdisks. ${ }^{14}$ 
Alloying Ge with Sn has also emerged as a viable means for creating a direct bandgap group IV material. Similar to tensile strain, increasing Sn content causes a transformation to direct bandgap. ${ }^{15}$ There have now been multiple demonstrations of lasing with $\mathrm{GeSn}^{16-18}$ up to a record high temperature of $230 \mathrm{~K}$, with $\mathrm{Sn}$ concentrations $\sim 16 \%$. ${ }^{19}$ Despite the huge success of GeSn alloying, a moderately direct band-structure is not sufficient for room temperature lasing, as electrons can thermally escape the $\Gamma$-valley and scatter into the L-valley, which has a much higher density of states. The $\Gamma$-valley has to be significantly lower in energy (several times $\left.k_{B} T\right)$ to prevent this effect. This is problematic as the high Sn concentration layers required for such a $\Delta E_{\mathrm{L}-\Gamma}$ result in challenging material growth, low thermal stability, and a high defect density that reduces the carrier lifetime.

It is, therefore, likely that a combination of external strain engineering and $\mathrm{Sn}$ alloying is required to achieve room temperature lasing, in order to improve the level of "directness," i.e., $\Delta E_{\mathrm{L}-\Gamma}$, while keeping thermal budgets sufficiently high for Si foundry processing, and potentially improving gain due to the strain induced splitting of the valence band. ${ }^{20-22}$ The use of SiN stressors for strain engineering GeSn is highly applicable, ${ }^{23,24}$ as the microbridge approach is not straightforward due to GeSn layers that are compressively strained as grown.

To fully understand the potential modal gain available in SiN strain engineered $\mathrm{Ge} / \mathrm{GeSn}$ devices, it is crucial to have an accurate measurement of the strain field in the devices' cross section, with high spatial resolution. This is not achievable with measurements such as Raman spectroscopy, which only probes the top plane, averaged over the penetration depth of the excitation source. As an alternative, the use of the transmission electron microscope (TEM) provides an instrument in which spatial resolutions of a few nanometers are routinely achieved, and in which diffraction-based techniques could provide suitable tools for strain measurement and spatially-resolved mapping. Here, we demonstrate the use of a precession electron diffraction (PED) technique to produce a crosssectional strain map of a SiN strained Ge microdisk and compare results to finite element modeling (FEM) of the strain field. The results are in good agreement with the modeling, and the previously measured optical properties of the structure, but also reveal the strain field around threading dislocations, and the resulting deviations from the simplified model, which does not include defects and dislocations.

\section{A. High spatial resolution strain measurement in the electron microscope}

Strain measurements are critical to understand and optimize the properties of the materials for improved device applications. It is, therefore, indispensable to have a method that can quantify the strain with nanoscale resolution and high precision. A number of methods to investigate strain in semiconductors have been employed. Early studies used X-ray diffraction ${ }^{25,26}$ and Raman spectroscopy. ${ }^{27-29}$ These techniques are now well-established and routinely have a spatial resolution of hundreds of nanometers.

As an alternative, the use of the transmission electron microscope (TEM) provides an instrument in which spatial resolutions of a few nanometers are routinely achieved, and in which diffraction-based techniques could provide suitable tools for strain measurement and spatially-resolved mapping. Béché et al. have recently compared some of the methods for strain mapping in $\mathrm{TEM}^{30}$ and these will be briefly summarized below.

Geometrical phase analysis (GPA) of atomic-resolution TEM or scanning TEM (STEM) images ${ }^{31-33}$ provides good spatial resolution but poor precision. ${ }^{34,35}$ Additionally, since this only works if there is a good quality atomic-resolution image, this does not work well for large fields of view, as crystal tilts and sample bending, together with changes in sample thickness, mean that this will never be achieved for the micrometer scale areas analyzed in this work.

Convergent beam electron diffraction $(\mathrm{CBED})^{36}$ has been successfully used to make strain measurements in semiconductor devices. The most important limitation of this methodology for strain analysis in crystal materials, however, is that reliable pattern simulations are required to fit with experimental results, which are time-consuming and model-dependent. ${ }^{37}$ This is not applicable for mapping of large areas and will not work with complex strain fields that tilt the crystal orientation away from a major zone axis.

Dark-field electron holography (DFEH) was first employed by Hÿtch et al. ${ }^{38}$ for strain measurement in electronic devices in 2008. Geometric phase measurements of the hologram (an interference pattern obtained from strained and unstrained region) were employed to map the strain at the nanoscale, ${ }^{30,31,39}$ which allows maps to be calculated with a high spatial resolution of up to $1 \mathrm{~nm}$ and a precision of $\pm 0.02 \% .^{35,38,40} \mathrm{~A}$ major restriction is that the sample should have a uniform thickness over the entire analyzed area $^{41}$ and an unstrained reference region of crystal must lie next to the region of interest, which is not always possible in real devices. ${ }^{42}$ Boureau et $a l^{40}$ achieved a field of view of $300 \mathrm{~nm}$, which is still not nearly enough for the large, micrometer-scale devices analyzed in this work.

In nanobeam electron diffraction (NBED), local diffraction patterns at each point are acquired by scanning an electron probe $(\sim 5-10 \mathrm{~nm})$ across a specimen. These diffraction patterns are compared with a reference pattern in order to provide relative shifts in the diffraction spots to quantify the deformation in the specimen. ${ }^{43-45}$ Though a precision of roughly $\pm 0.1 \%$ can be achieved with this technique, results can be erroneous and noisy at times. This occurs due to the contrast within diffraction spots arising from multiple scattering processes (dynamical effects). ${ }^{46}$ Also, to improve the spatial resolution, a larger convergence angle is required, which makes the diffraction spots into large disks which are even more prone to dynamical scattering within the disks.

To overcome these hurdles with NBED, a method was developed of precessing the electron beam at each point on the specimen while acquiring the diffraction pattern. ${ }^{47}$ Thus, the intensity at each diffraction spot is integrated from many incident angles, which averages out most of the dynamical effects within the spots, creating small disks of uniform intensity, allowing their automated detection and locations with subpixel accuracy. Most importantly, precession also enables the collection of higher-order reflections, which are more sensitive than lower order reflections to small changes in lattice parameters. This technique is known as Scanning Precession Electron Diffraction (SPED). For an application like the present one, this is the ideal technique-it can be applied in 
relatively thick materials $(\sim 100 \mathrm{~nm})$, has a resolution of a few nanometers (mainly limited by beam spreading in a thicker sample), can be performed along or near a major zone axis, and has a high precision with a sufficiently large precession angle to include higher index spots, ${ }^{48,49}$ and most importantly, it can be performed over very large sample areas running into several micrometers (mainly limited by the capabilities of the scanning system in the scanning TEM). The ability to use relative thick specimens helps to reduce strain relaxation and maintain bulklike strain fields in the plane of the sample, as observed previously in Farooq et al. ${ }^{50}$ Though the use of SPED for strain measurement is relatively new, it has already been employed to study strain in SiGe and Ge based semiconducting nanostructures and devices. ${ }^{48,49,51-54}$

In this paper, we report on high precision SPED measurements to accurately map the strain in a microdisk-shaped Ge structure in which the strain is concentrated in a small region. These experimental measurements are then compared with finite element modeling (FEM) to evaluate the strain, and the differences are highlighted, and the microstructural reasons for these differences are investigated using conventional TEM techniques. Our work demonstrates the high potential of the SPED technique for the investigation of inhomogeneously strained microstructures with a prospect of applications in future photonics.

\section{EXPERIMENTAL DETAILS}

\section{A. Fabrication of a Ge-microdisk structure}

Low-Energy Plasma-Enhanced Chemical Vapor Deposition (LEPECVD) was used to grow a $380 \mathrm{~nm}$ layer of Ge heterostructure on top of a $\mathrm{Si}(001)$ substrate at a low temperature of $500{ }^{\circ} \mathrm{C} .{ }^{55}$ The Ge structure was patterned by electron beam lithography in a Vistec VB6 tool using hydrogen silsesquioxane (HSQ) resist and was dry etched afterwards in a mixed $\mathrm{SF}_{6}$ and $\mathrm{C}_{4} \mathrm{~F}_{8}$ recipe through to the Si layer. ${ }^{56} \mathrm{Si}$ was anisotropically wet etched to undercut the structure to leave a controlled size of Si post to support the disk, using tetramethylammonium hydroxide (TMAH) and isopropyl alcohol (IPA). This was followed by the coating of the structures with high stress silicon nitride in an inductively coupled plasma enhanced chemical vapor deposition (ICP-PECVD) tool. This produces a high compressive stress of $\sim 2.4 \mathrm{GPa}$ in the film, controlled through the deposition parameters. ${ }^{57}$ More details of growth can be found in Millar et al. ${ }^{13}$

\section{B. Microscopy specimen preparation}

A cross-sectional sample was prepared using a focused ion beam (FIB, FEI Nova Nanolab 200) lift-out procedure ${ }^{58,59}$ to produce a thin lamella of a relatively constant thickness for STEM analysis. After the identification of a suitable area for crosssectioning a microdisk, a thin platinum (Pt) layer was deposited using electron beam deposition to protect the cross-sectional specimen from $\mathrm{Ga}^{+}$ion beam induced damage (Pt deposition should not stress the structure any further). Trenches were then created on both sides and parallel to the structure to create a $1 \mu \mathrm{m}$ thick and $15 \mu \mathrm{m}$ wide lamella. This was carefully lifted out in situ using the micromanipulator tip and attached to an Omniprobe support grid. Finally, FIB milling and polishing were carried out on both sides of
TEM lamella, using a reduced beam current, to produce a nearly parallel sided specimen of a final thickness of about $100 \mathrm{~nm}$ [the sample was left a little thicker than some (S)TEM specimens used for atomic-resolution analysis, in order to leave more bulklike strain states within].

\section{Precession electron diffraction and imaging}

SPED was performed using a $200 \mathrm{kV}$ CM200 (S)TEM equipped with a field emission gun (FEG) operated in the STEM mode and using a convergence half angle of $0.7 \mathrm{mrad}$. Precession of the electron beam was performed at an angle of $1.8^{\circ}$ and a frequency of $100 \mathrm{~Hz}$ using a NanoMEGAS DigiSTAR unit. The probe size was $\sim 10 \mathrm{~nm}$ for the chosen condenser aperture of $20 \mu \mathrm{m}$. The step size was $10 \mathrm{~nm}$ for the strain maps, and the datasets were recorded with an acquisition time of $0.1 \mathrm{~s}$ per pattern using a standard Stingray optically-coupled camera (which images the small focusing screen in the TEM).

The reference diffraction pattern, obtained from an unstrained region, was modified numerically to make it comparable with each diffraction pattern taken from a strained region. This does not require identifying individual diffraction spots and thus makes it easier to derive strain coefficients from processing these diffraction patterns. Thus, 2D maps for in-plane strain $\varepsilon_{x x}$, out-of-plane strain $\varepsilon_{z z}$, and shear strain $\varepsilon_{x z}$ are obtained. ${ }^{49,54}$ The lattice strains were originally evaluated with respect to the Si lattice parameter measured on the pedestal and, therefore, includes the lattice misfit of $\sim 4.2 \%$ that exists between $\mathrm{Ge}$ and $\mathrm{Si}$, but are corrected to refer to strains according to the lattice parameter of unstrained Ge. This can be accomplished using the equation

$$
\varepsilon_{\mathrm{Ge} \text { reference }}=\frac{a_{\mathrm{Si}}}{a_{\mathrm{Ge}}}\left(\varepsilon_{\mathrm{Si} \text { reference }}+1\right)-1,
$$

where $\varepsilon_{\mathrm{Si} \text { reference }}$ is the strain determined using the silicon substrate as a reference, $\varepsilon_{\mathrm{Ge}}$ reference is the strain with unstrained germanium as a reference, and $a_{\mathrm{Si}}$ and $a_{\mathrm{Ge}}$ are the lattice parameters of unstrained silicon and germanium, respectively. Example diffraction patterns from different areas of the structure are shown in Fig. S1 in the supplementary material.

In order to proceed with crystalline orientation identification, thousands of simulated electron diffraction spot patterns (so-called templates) were utilized for each crystallographic phase in the sample. Local crystal orientations were obtained by comparing experimentally acquired patterns using cross-correlation matching techniques with the template database. ${ }^{60}$

STEM imaging of the structure was undertaken on a probe corrected JEOL ARM $200 \mathrm{~F}$ equipped with a cold field emission gun operated at $200 \mathrm{kV}$ in the STEM mode and using a convergence half angle of $29 \mathrm{mrad}$. High angle annular dark-field (HAADF) imaging was performed with an inner detector angle of $107 \mathrm{mrad}$. At this angle, the contrast is strongly dependent on the atomic number and, therefore, the image brightness is strongly correlated to chemistry. A Gatan GIF Quantum ER energy filter/spectrometer with fast Dual EELS (electron energy loss spectroscopy) was used to record spectrum images (SIs) with a collection half angle of $36 \mathrm{mrad}$ and a dispersion of $1.0 \mathrm{eV}$ per channel, to 
determine the final thickness of the lamella using the $t / \lambda$ method. Conventional dark-field TEM imaging was performed on a FEI Tecnai T20 operated at $200 \mathrm{kV}$ in order to view dislocations in the Ge microdisk and in an equivalent specimen constructed from a Ge heterolayer grown in an identical way but not fabricated into a strained microdisk.

\section{Finite element simulations}

Finite element simulations ${ }^{61}$ have been used to generate strain maps which have been compared with experimental results. A three-dimensional FEM model was employed in COMSOL Multiphysics, taking into account the anisotropy of the Ge elasticity tensor and using the geometry of the $4 \mu \mathrm{m}$ microdisk measured by STEM (shown in Fig. 1). The model also includes the $2.45 \mathrm{GPa}$ silicon nitride stressor, which was optimized to make the vertical displacement of the microdisk edge in the model comparable to that measured by STEM in Fig. 1. The vertical deflection at the microdisk edge is $\sim 150 \mathrm{~nm}$. The model has the $\mathbf{x}$ and $\mathbf{z}$ directions oriented along two $\langle 100\rangle$ crystallographic directions, which also represent the edge of the microdisk.

\section{RESULTS AND DISCUSSIONS}

\section{A. Strain mapping}

Figure 1(a) shows an overview image of a FIB section through a $4 \mu \mathrm{m}$ diameter Ge microdisk acquired using HAADF STEM. Different layers in the sample can be distinguished by the layer brightness and are labeled accordingly in the figure. The image shows a high-quality cross section with no "curtaining" or
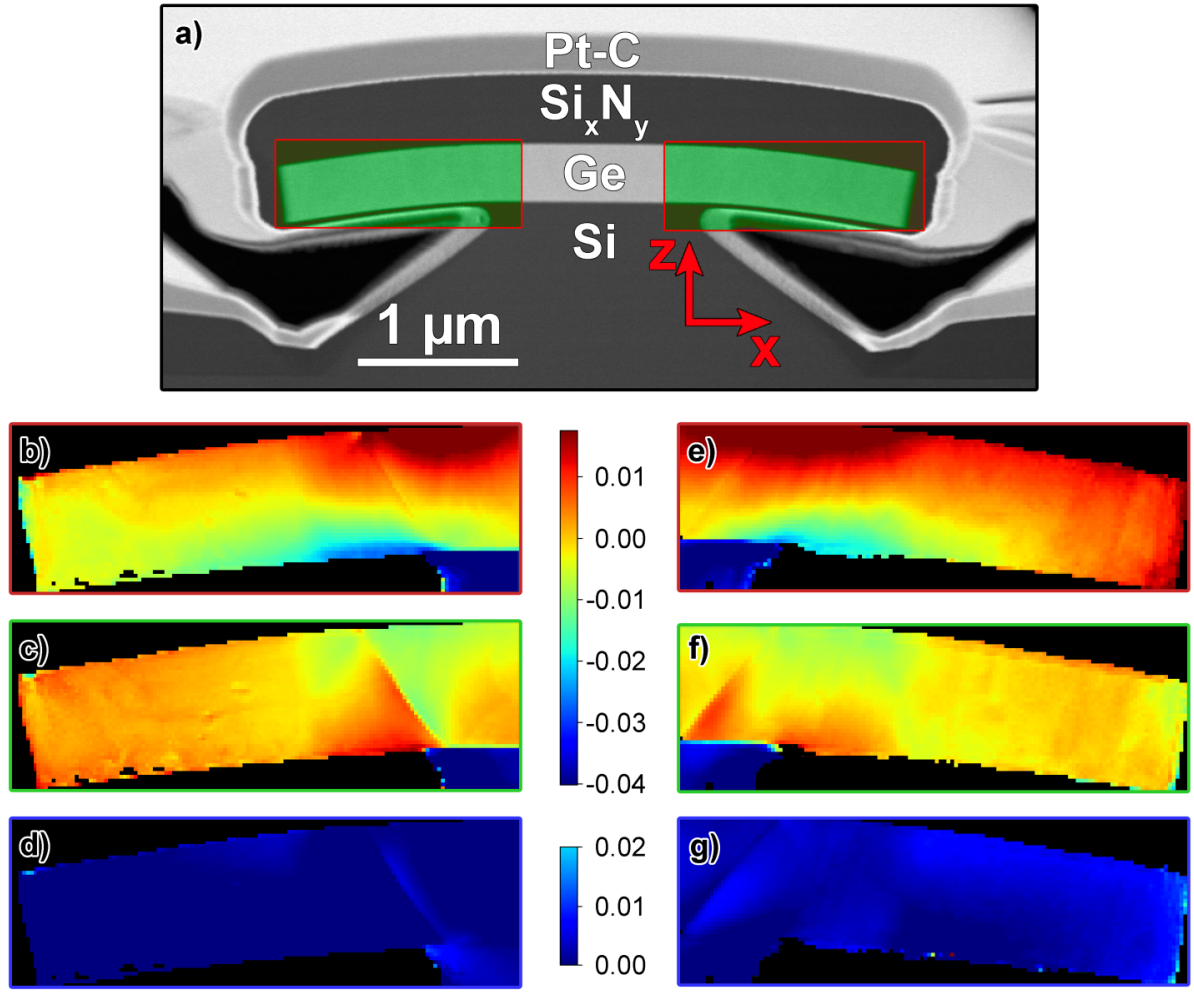

$-0.01$

-0.02 f)

$-0.03$

$-0.04$
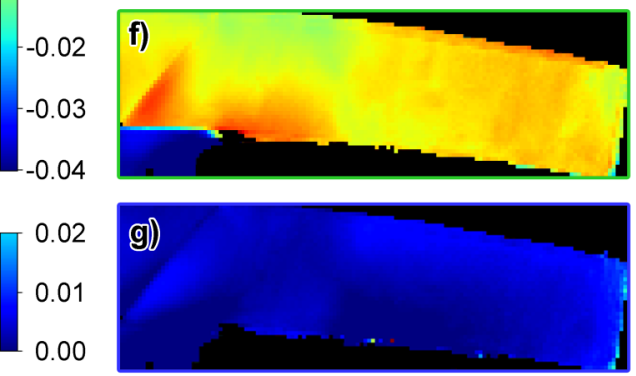

h)

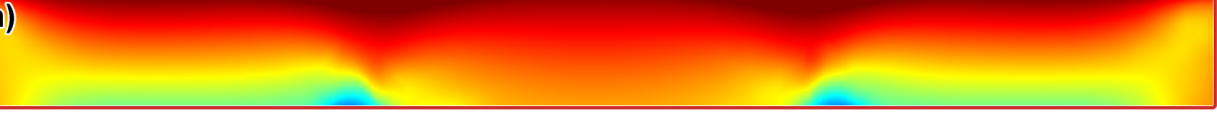

i)

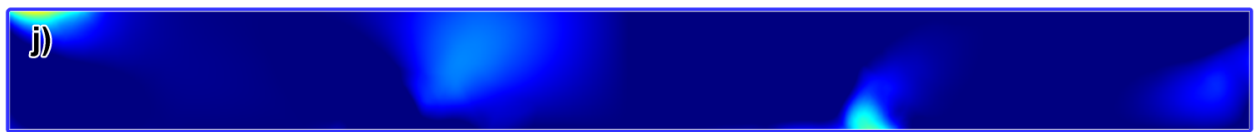

FIG. 1. Images and strain maps (referenced to unstrained $\mathrm{Ge}$ ) of a $4 \mu \mathrm{m} \mathrm{Ge}$ microdisk structure grown on a Si post, with SiN layer as a stressor on top: ${ }^{13}$ (a) a HAADF STEM image of the FIB-prepared TEM cross section, protective platinum layers deposited during cross-sectioning in the FIB are also visible. Rectangular areas shaded in green have been used for strain mapping using PED. The coordinate system chosen is also shown. (b)-(d) Strain maps acquired by precession diffraction for the $\varepsilon_{\mathrm{xx}}, \varepsilon_{\mathrm{zz}}, \varepsilon_{\mathrm{xz}}$, in the left wing of the Ge microdisk, and (e)(g) represents the same maps extracted for the right wing of the microdisk. The colour bars show the relationship between colour and strain level. (h)-(j) show 2D slices from finite element models through a $4 \mu \mathrm{m} \mathrm{Ge}$ microdisk demonstrating the in-plane strain components, $\varepsilon_{\mathrm{xx}}, \varepsilon_{\mathrm{zz}}$, and $\varepsilon_{\mathrm{xz}}$. The same colour scales apply as for the experimental strain maps. 
preferential milling artefacts, and the outer wings are clearly slightly bent, as expected. Experimental measurements using SPED have been performed in the outer parts (wings) of the crystalline Ge; marked red in Fig. 1(a). The axes chosen are drawn and the y axis is the direction of electron beam and lies normal to the image.

Figures $1(\mathrm{~b})-1(\mathrm{~g})$ show the experimental strain maps acquired by PED for both wings for in-plane $\varepsilon_{x x}$, out-of-plane $\varepsilon_{z z}$, and shear $\varepsilon_{x z}$ components. The red areas represent the positive (tensile) strain, whereas the blue areas (especially the $\mathrm{Si}$ ) represent the negative (compressive) strain for $\varepsilon_{x x}$ and $\varepsilon_{z z}$ maps. At the top of $\mathrm{Ge}$ around the edges of the post, the $\varepsilon_{x x}$ strains are tensile of just below $2 \%$ but the $\varepsilon_{z z}$ values in the same regions are slightly compressive, about $1 \%$ below the Ge lattice parameter. At the base of the wings next to the post, this trend is reversed and $\varepsilon_{x x}$ is compressive and $\varepsilon_{z z}$ is tensile. Away from the post, there are smaller variations in $\varepsilon_{z z}$ but the trend of $\varepsilon_{x x}$ being tensile at the top and compressive at the bottom continues. There are also some sharp changes in the vertical strain seen in straight diagonal lines in Figs. 1(c) and 1(f). Both of these radiate from a point close to the corner of the post and suggest a breakdown in the idealized strain state that would be predicted by continuum elastic considerations. Moreover, the decrease in the strain observed along a vertical line from top to bottom of the microdisk depends upon the diameter of the post and can vary accordingly. ${ }^{62}$ Shear strains are relatively small in all cases [Figs. 1(d) and 1(g)].

The best fit FEM of the strains in a slice through the center of the Ge microdisk structure is shown in Fig. 1(h) for $\varepsilon_{x x}$, Fig. 1(i) for $\varepsilon_{z z}$, and Fig. 1(j) for $\varepsilon_{x z}$, using the same color scale in absolute terms. The $\varepsilon_{x x}$ map shows a maximum value of $\sim 2.1 \%$ at the top. This is slightly above the experimental measurements of the peak strain of $1.8 \%$ but certainly consistent (especially when there may have been strain relaxation in the real microdisks, as shown below). Additionally, the simulations predict a minimum value of $\varepsilon_{x x-}$ $\sim-0.5 \%$ at the bottom near the interface, and this is in close agreement with the experimental value of $-0.6 \%$. Raman measurements were done previously on this sample to investigate the strain components. ${ }^{13}$ Raman measurements were consistent with a peak in-plane strain $\varepsilon_{x x}$ of $>2 \%$ which also agrees with the experimental and simulation results presented here. Thus, it is confirmed that the $\mathrm{SiN}$ stressor produces a strain of the appropriate magnitude to explain the optical and mechanical properties demonstrated in our previous work. ${ }^{13}$ The experiments, however, only show this peak stress just outside the radius of the post, whereas simulations show it extending almost to the edge. This probably happens due to stress relaxation within the microdisk due to plastic deformation. This means that the sample would have a ring-shaped region of peak strain at which the effects would be most significant.

There is a reasonable qualitative match between the $\varepsilon_{z z}$ simulation of Fig. 1(i) and the maps in the area on the post and just outside it, although the strain is more relaxed at the outer edges, as observed for the $\varepsilon_{x x}$ strain. The shear strains are small, and though there may be some similarities in general tendencies, exact matching is difficult, and the magnitudes of any real shear strains are clearly less than in the FEM.

FEM has certain limitations which restrict the exact agreement of the model with the experimental results. Firstly, PED measurements were performed on a thin lamella prepared from the bulk structure. While significant strain relaxation is not expected in-plane due to the specimen thickness, it is possible that there will be some relaxation near the surfaces in ways that alter the results from the stress distribution in the bulk. ${ }^{63}$ Moreover, other work by one of the authors has demonstrated that the strain state in a relatively thick TEM sample (as used here) is a reasonable approximation to bulk results from X-ray diffraction. ${ }^{50}$ Moreover, any surface relaxation does not inhibit the observation of trends in the strain field that are consistent with the FEM models. Secondly, the epitaxy in the real sample is far more complex than the simplified model chosen in this FEM model. Specifically, it does not include factors such as any deviation from perfect epitaxy, and does not include dislocations and plastic deformation, so this could be the cause for any discrepancy between simulated and experimental strain maps. To further investigate these effects, dark-field imaging and orientation mapping were performed.

\section{B. Dark-field imaging}

Dark-field images have been recorded for the specimen using two-beam conditions for various diffraction vectors to reveal dislocations. Some representative images are shown in Fig. 2 where wellspaced threading dislocations are identifiable in various parts of the Ge structure as bright lines - similar threading dislocations were also observed in nominally identical Ge heterolayers without any fabrication steps. Additionally, dense arrays of dislocations in slip planes are visible marked by arrows emanating from the Si-Ge interface

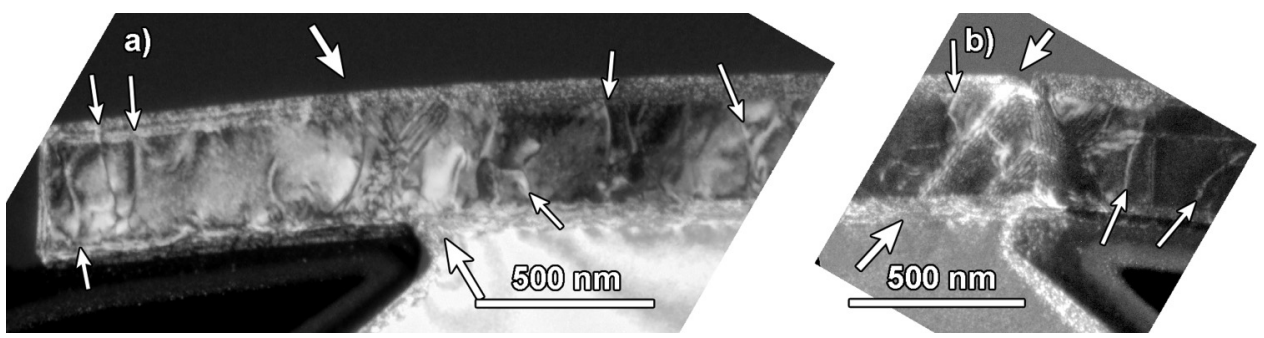

FIG. 2. Dark-field images taken across the Ge microdisk. Several dislocations are seen with significant changes in contrast from the background, some may have been threading dislocations from original growth. The arrows indicate slip traces along which a larger number of dislocations moved resulting in a larger misorientation. (a) the left wing of Figs. 1(b)-1(d); (b) detail of the right wing of Figs. 1(e)-1(g). 
close to the outside edge of the post. These clearly show that plastic deformation by dislocation slip has occurred as a consequence of the stress concentration around these sharp corners. This was exactly the same sample as for the strain mapping in Fig. 1, and the slip plane locations correspond to the sharp changes in $\varepsilon_{\mathrm{zz}}$ in those strain maps. In some samples, cracks have been observed at the outside edge of these slip planes (i.e., regions of high tensile strain).

The threading dislocation density (TDD) was calculated by the line-intercept method ${ }^{64}$ which has been able to provide reliable results for dislocation density measurements ${ }^{65}$ using TEM images. In this method, randomly oriented lines are drawn through dislocations, over TEM images, and the dislocations are then marked with points. The dislocation density $\rho$ is given by the number of points $N$ divided by the total line length of the random lines $L_{r}$, multiplied by the thickness $t$, i.e., $\rho=\frac{2 N}{t L_{r}}$. The method strictly relies upon the accurate measurement of the thickness which in the present study was found by means of electron energy loss spectroscopy using a mean free path of $97 \mathrm{~nm}$ calculated by the Iakoubovskii method ${ }^{66}$ after correction by a factor of 0.8 , as in the previous work. ${ }^{67}$ The mean density across the entire microdisk was found to be $1.48 \pm 0.4 \times 10^{10} \mathrm{~cm}^{-2}$. This is rather greater than that in the raw unstrained $\mathrm{Ge}$ grown on $\mathrm{Si}$, prior to the fabrication of the disks, which is $1.6 \pm 0.5 \times 10^{9} \mathrm{~cm}^{-2}$. Example dark-field TEM images of dislocations in the layer before patterning are shown in Fig. S2 in the supplementary material. The threading dislocation networks and density in the outer part of the microdisk, however, appear similar to those in the unstrained material, and the main reason for the increased dislocation density in the microdisks is slip and dislocation multiplication close to the sharp corner at the end of the undercut, as noted above. It should be noted that the Ge epitaxial layer used here did not receive cyclic annealing to reduce the TDD, to avoid diffusion and clustering of the phosphorous doping. ${ }^{68}$ Cyclic annealing of comparable layers can be used to reduce TDD down to $\sim 1 \times 10^{7} \mathrm{~cm}^{-2}$.

\section{Orientation mapping}

The crystal orientation should vary with position due to the bending resulting from the SiN stressor, and this can also be investigated from the SPED datasets used for the strain mapping, as previously performed by Estradé et al. ${ }^{53}$ The $\mathbf{c}$ axis orientation map is displayed in Fig. 3(a) and can be interpreted using the color code shown [this shows the angle of the $\mathbf{c}$ axis of the crystal to the $\mathbf{z}$ axis of the figure, as defined in Fig. 1(a)]. The color of each point in the map designates the crystallographic orientation of the $\mathbf{c}$ axis of the crystal at that point with respect to the vertical, according to the color key provided. This tilt angle as a function of position along a trace from left to right through the center of the microdisk is plotted in Fig. 3(b). This shows that the central portion of the microdisk is basically oriented parallel with the silicon. The orientation changes most rapidly immediately outside the center post. There may be slight discontinuities at the edges of the post, which could possibly correspond to the slip traces and dislocation pileups seen in Fig. 2, but the angular resolution in precession electron diffraction is rather too poor (limited to about $0.5^{\circ 70}$ ) to be certain about whether the slip traces cause significant crystal tilts. Further away from the center post, the curvature of the crystal becomes

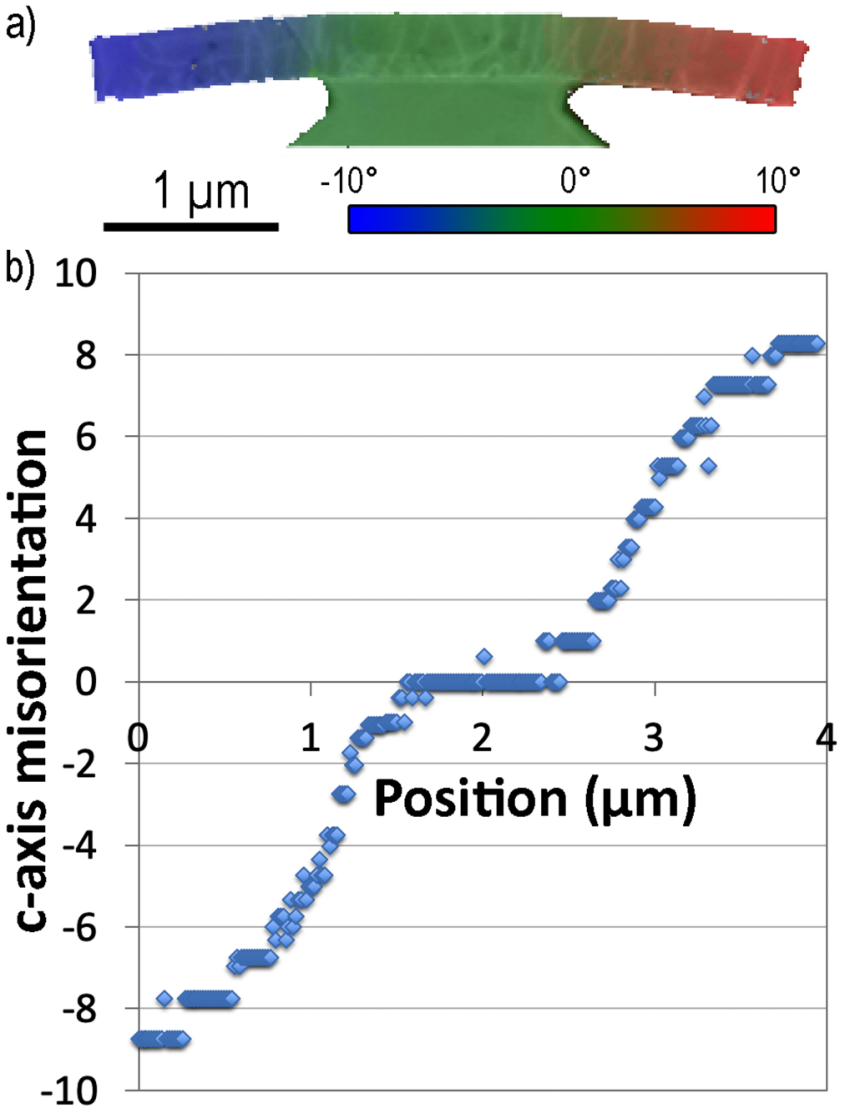

FIG. 3. (a) A map of the orientation of the [001] axis to the vertical in the Ge microdisk on $\mathrm{Si}$ (superimposed on the pattern quality map, which shows some variations in contrast due to dislocations), (b) the misorientation profile of the $c$ axis of the crystal structure to the vertical as a function of position going from left to right across a scan of the whole $4 \mu \mathrm{m}$ microdisk.

gentler, which corresponds to the reduced strains measured in the outer parts of the wings in Fig. 1. Nevertheless, the overall trend in orientation with position is much as expected and corresponds with the observed stress levels, as the positions of highest stress in Fig. 1 are also the areas of highest curvature in Fig. 3(b).

\section{General discussion}

In this work, we have analyzed a tensile strained Ge microdisk structure fabricated on $\mathrm{Si}$, which has been tensile strain engineered with an external SiN stressor layer. Peak strain levels are found to be consistent with experimental measurements in Ref. 23, which included micro-Raman and photoluminescence (PL). These structures demonstrated huge red-shifts in PL $(\sim 450 \mathrm{~nm})$ and large shifts in the Raman spectra produced by the longitudinal optical phonon. Both of these techniques were consistent with a biaxial tensile strain of $\sim 2 \%$. The discrepancies here are likely the result of some strain relaxation after the FIB preparation, coupled with relaxation surrounding threading dislocations, especially the slip planes. 
The strain-field results presented here are in close agreement with expectations from FEM, as was previously seen in PED-FEM comparisons of $(\mathrm{Al}, \mathrm{Ga}) \mathrm{N}$ heterostructures by Reisinger. $^{71}$ Nevertheless, there is clear evidence that some loss of elastic strain has occurred through dislocation motion and multiplication at the highest stress concentrations around the corners of the Si supporting posts. This, therefore, demonstrates that such techniques are extremely applicable to such strain engineered photonic structures and could be used to determine and analyze modal gain. This is particularly useful when the presence of dislocations may cause deviation from the simplified finite element models.

It is clear from this work that the single stressor layer leads to a large inhomogeneity across the microdisk, which is undesirable for gain in Ge/GeSn structures. ${ }^{72}$ It is, however, interesting to note that such strain engineering could be highly applicable to step graded GeSn layers. Step grading has emerged as an approach for minimizing dislocations in the active layer (highest Sn content) layer, which is grown on top of multiple layers of lower Sn content, which serve as virtual substrates. Such photonic structures have been fabricated into microdisks and Fabry-Pérot cavities and have demonstrated lasing. ${ }^{16,17,19}$ In such devices, the highest Sn concentration layers have the highest refractive index, meaning that the optical mode is pulled slightly towards the top portion of the microdisk, where the mode will overlap more with the tensile strain field. The lower, compressively strained portion of the microdisk could comprise either Ge, SiGeSn, or low Sn content GeSn buffers that do not contribute to modal gain, and are, therefore, not detrimentally affected by compressive strain at the bottom of the microdisk. In particular, higher-order modes may overlap more significantly with the highest tensile strained regions of the microdisk; however, a modal gain analysis is beyond the scope of this work. This work does, however, demonstrate the applicability of PED techniques to analyze strain fields in such photonic structures, where FEM models have previously been required to calculate optical overlap with the strain field. ${ }^{71}$

\section{CONCLUSION}

We have successfully characterized a Ge microdisk structure, grown on $\mathrm{Si}$ by LEPECVD, using strain and misorientation mapping by scanning precession electron diffraction (SPED) with high accuracy and spatial resolution. Experimentally acquired strain maps for the $\varepsilon_{x x}, \varepsilon_{x z}$, and $\varepsilon_{z z}$ components have been compared with FEM simulations. The peak strains are also consistent with those inferred from Raman scattering measurements in the previous work. While the spatial variation of the strain and the magnitudes of peak strains match well between experiment and simulation, some discrepancies are present. Specifically, there are sharp discontinuities in strain across diagonal bands emanating from close to the corner of the Si pillar and the Ge microdisk above, which appear to correspond to dislocation pileups along slip traces. This demonstrates that the observed peak strain of $2.1 \%$ tensile strain at the top surface is close to the maximum that can be sustained in Ge before plastic deformation and possible cracking and failure of the structure. Nevertheless, it also demonstrates that a certain level of plastic deformation does not prevent the formation of large enough tensile strains to produce the significant Raman shifts observed in our previous work.

\section{SUPPLEMENTARY MATERIAL}

See the supplementary material for example diffraction patterns from different areas in the structure in the precession electron diffraction dataset and for dark-field images of threading dislocations in Ge layers prior to patterning into microdisks.

\section{ACKNOWLEDGMENTS}

A. Bashir is thankful to the Schlumberger Foundation Faculty for the Future program for the provision of a research fellowship. We are grateful to SUPA and the University of Glasgow for the provision of the JEOL ARM200F microscope used in this work. We are also grateful to Dr. Stavros Nicolopoulos from NanoMegas for providing access to the scanning precession electron diffraction used in this work for the strain mapping. The work was supported by the Engineering and Physical Sciences Research Council (EPSRC) project (No. EP/N003225/1) and the European Union 7th Framework Programme project GEMINI (No. 613055). R.W.M. is grateful to the UK Royal Academy of Engineering for the provision of a Research Fellowship (Project RF/201819/18/187).

\section{REFERENCES}

${ }^{1}$ Y. Song, H. Zhou, Q. Xu, J. Luo, H. Yin, J. Yan, and H. Zhong, J. Electron. Mater. 40, 1584 (2011).

${ }^{2}$ Y. Kang, H.-D. Liu, M. Morse, M. J. Paniccia, M. Zadka, S. Litski, G. Sarid, A. Pauchard, Y.-H. Kuo, H.-W. Chen, W. S. Zaoui, J. E. Bowers, A. Beling, D. C. McIntosh, X. Zheng, and J. C. Campbell, Nat. Photonics 3, 59-63 (2009).

${ }^{3}$ Y.-H. Kuo, Y. K. Lee, Y. Ge, S. Ren, J. E. Roth, T. I. Kamins, D. A. B. Miller, and J. S. Harris, Nature 437, 1334-1336 (2005).

${ }^{4}$ J. E. Roth, O. Fidaner, R. K. Schaevitz, Y.-H. Kuo, T. I. Kamins, J. S. Harris, and D. A. B. Miller, Opt. Express 15, 5851-5859 (2007).

${ }^{5}$ R. E. Camacho-Aguilera, Y. Cai, N. Patel, J. T. Bessette, M. Romagnoli, L. C. Kimerling, and J. Michel, Opt. Express 20, 11316-11320 (2012).

${ }^{6}$ J. F. Liu, X. C. Sun, R. Camacho-Aguilera, L. C. Kimerling, and J. Michel, Opt. Lett. 35, 679-681 (2010).

${ }^{7}$ R. Koerner, M. Oehme, M. Gollhofer, M. Schmid, K. Kostecki, S. Bechler, D. Widmann, E. Kasper, and J. Schulze, Opt. Express 23, 14815-14822 (2015).

${ }^{8}$ M. R. Barget, M. Virgilio, G. Capellini, Y. Yamamoto, and T. Schroeder, J. Appl. Phys. 121, 245701 (2017).

${ }^{9}$ M. El Kurdi, M. Prost, A. Ghrib, S. Sauvage, X. Checoury, G. Beaudoin, I. Sagnes, G. Picardi, R. Ossikovski, and P. Boucaud, ACS Photonics 3, 443-448 (2016).

${ }^{10}$ M. J. Süess, R. Geiger, R. A. Minamisawa, G. Schiefler, J. Frigerio, D. Chrastina, G. Isella, R. Spolenak, J. Faist, and H. Sigg, Nat. Photonics 7, 466 (2013).

${ }^{11}$ D. S. Sukhdeo, D. Nam, J.-H. Kang, M. L. Brongersma, and K. C. Saraswat, Photonics Res. 2, A8-A13 (2014).

${ }^{12}$ S. Bao, D. Kim, C. Onwukaeme, S. Gupta, K. Saraswat, K. H. Lee, Y. Kim, D. Min, Y. Jung, H. Qiu, H. Wang, E. A. Fitzgerald, C. S. Tan, and D. Nam, Nat. Commun. 8, 1845 (2017).

${ }^{13}$ R. W. Millar, K. Gallacher, J. Frigerio, A. Ballabio, A. Bashir, I. MacLaren, G. Isella, and D. J. Paul, Opt. Express 24, 4365-4374 (2016).

${ }^{14}$ A. Elbaz, M. El Kurdi, A. Aassime, S. Sauvage, X. Checoury, I. Sagnes, C. Baudot, F. Boeuf, and P. Boucaud, APL Photonics 3, 106102 (2018).

${ }^{15}$ S. Wirths, R. Geiger, N. von den Driesch, G. Mussler, T. Stoica, S. Mantl, Z. Ikonic, M. Luysberg, S. Chiussi, J. M. Hartmann, H. Sigg, J. Faist, D. Buca, and D. Grützmacher, Nat. Photonics 9, 88-92 (2015). 
${ }^{16}$ J. Margetis, S. Al-Kabi, W. Du, W. Dou, Y. Zhou, T. Pham, P. Grant, S. Ghetmiri, A. Mosleh, B. Li, J. Liu, G. Sun, R. Soref, J. Tolle, M. Mortazavi, and S.-Q. Yu, ACS Photonics 5, 827-833 (2018).

${ }^{17}$ V. Reboud, A. Gassenq, N. Pauc, J. Aubin, L. Milord, Q. M. Thai, M. Bertrand, K. Guilloy, D. Rouchon, J. Rothman, T. Zabel, F. Armand Pilon, H. Sigg, A. Chelnokov, J. M. Hartmann, and V. Calvo, Appl. Phys. Lett. 111, 092101 (2017).

${ }^{18}$ D. Stange, N. von den Driesch, T. Zabel, F. Armand-Pilon, D. Rainko, B. Marzban, P. Zaumseil, J.-M. Hartmann, Z. Ikonic, G. Capellini, S. Mantl, H. Sigg, J. Witzens, D. Grützmacher, and D. Buca, ACS Photonics 5, 4628-4636 (2018).

${ }^{19}$ Q. M. Thai, N. Pauc, J. Aubin, M. Bertrand, J. Chrétien, V. Delaye, A. Chelnokov, J.-M. Hartmann, V. Reboud, and V. Calvo, Opt. Express 26, 32500-32508 (2018).

${ }^{20}$ S. Wirths, Z. Ikonic, A. T. Tiedemann, B. Holländer, T. Stoica, G. Mussler, U. Breuer, J. M. Hartmann, A. Benedetti, S. Chiussi, D. Grützmacher, S. Mantl, and D. Buca, Appl. Phys. Lett. 103, 192110 (2013).

${ }^{21}$ D. Rainko, Z. Ikonic, A. Elbaz, N. von den Driesch, D. Stange, E. Herth, P. Boucaud, M. El Kurdi, D. Grützmacher, and D. Buca, Sci. Rep. 9, 259 (2019).

${ }^{22}$ Y. Liu, C. Fang, X. Gao, G. Han, Q. Zhang, Y. Shao, J. Zhang, and Y. Hao, IEEE Photonics J. 10, 1-9 (2018)

${ }^{23}$ R. W. Millar, D. C. S. Dumas, K. F. Gallacher, P. Jahandar, C. MacGregor, M. Myronov, and D. J. Paul, Opt. Express 25, 25374-25385 (2017).

${ }^{24}$ C. S. Fenrich, X. Chen, R. Chen, Y.-C. Huang, H. Chung, M.-Y. Kao, Y. Huo, T. I. Kamins, and J. S. Harris, ACS Photonics 3, 2231-2236 (2016)

${ }^{25}$ I. Robinson and R. Harder, Nat. Mater. 8, 291-298 (2009).

${ }^{26}$ T. Vreeland and B. M. Paine, J. Vac. Sci. Technol. A 4, 3153-3159 (1986).

${ }^{\mathbf{2 7}} \mathrm{V}$. Senez, A. Armigliato, I. De Wolf, G. Carnevale, R. Balboni, S. Frabboni, and A. Benedetti, J. Appl. Phys. 94, 5574-5583 (2003).

${ }^{28}$ J. C. Tsang, P. M. Mooney, F. Dacol, and J. O. Chu, J. Appl. Phys. 75, 8098-8108 (1994).

${ }^{29}$ J. Greil, A. Lugstein, C. Zeiner, G. Strasser, and E. Bertagnolli, Nano Lett. 12, 6230-6234 (2012)

${ }^{30}$ A. Béché, J. L. Rouvière, J. P. Barnes, and D. Cooper, Ultramicroscopy 131, $10-23$ (2013).

${ }^{31}$ M. J. Hÿtch, E. Snoeck, and R. Kilaas, Ultramicroscopy 74, 131-146 (1998).

${ }^{32}$ F. Hüe, M. Hÿtch, H. Bender, F. Houdellier, and A. Claverie, Phys. Rev. Lett. 100, 156602 (2008).

${ }^{33}$ C.-Y. Wen, M. C. Reuter, D. Su, E. A. Stach, and F. M. Ross, Nano Lett. 15, 1654-1659 (2015).

34J. Chung, G. Lian, and L. Rabenberg, IEEE Electron Device Lett. 31, 854-856 (2010).

${ }^{35}$ D. Cooper, J.-P. Barnes, J.-M. Hartmann, A. Béché, and J.-L. Rouvière, Appl. Phys. Lett. 95, 053501 (2009).

${ }^{36}$ A. Armigliato, R. Balboni, G. P. Carnevale, G. Pavia, D. Piccolo, S. Frabboni, A. Benedetti, and A. G. Cullis, Appl. Phys. Lett. 82, 2172-2174 (2003).

${ }^{37}$ M. Kim, J. M. Zuo, and G.-S. Park, Appl. Phys. Lett. 84, 2181-2183 (2004).

${ }^{38}$ M. Hÿtch, F. Houdellier, F. Hüe, and E. Snoeck, Nature 453, 1086-1089 (2008).

${ }^{39}$ A. Béché, J. L. Rouvière, J. P. Barnes, and D. Cooper, Ultramicroscopy 111, 227-238 (2011)

${ }^{40} \mathrm{~V}$. Boureau, R. McLeod, B. Mayall, and D. Cooper, Ultramicroscopy 193, $52-63$ (2018)

${ }^{41}$ V. V. Hoang, Y. Cho, J. H. Yoo, S.-K. Hong, Y. H. Choi, S. Choi, W. Jung, C. K. Jeong, and J.-M. Yang, Microscopy 65, 499-507 (2016).

${ }^{42}$ D. Cooper, C. Le Royer, A. Béché, and J.-L. Rouvière, Appl. Phys. Lett. 100, 233121 (2012).
${ }^{43}$ A. Armigliato, S. Frabboni, and G. C. Gazzadi, Appl. Phys. Lett. 93, 161906 (2008).

${ }^{44}$ F. Uesugi, A. Hokazono, and S. Takeno, Ultramicroscopy 111, 995-998 (2011).

${ }^{45}$ A. Béché, J. L. Rouvière, L. Clément, and J. M. Hartmann, Appl. Phys. Lett. 95, 123114 (2009).

${ }^{46}$ D. Cooper, A. Béché, J. M. Hartmann, V. Carron, and J.-L. Rouvière, Semicond. Sci. Technol. 25, 095012 (2010).

${ }^{47}$ R. Vincent and P. A. Midgley, Ultramicroscopy 53, 271-282 (1994).

${ }^{48}$ J.-L. Rouvière, A. Béché, Y. Martin, T. Denneulin, and D. Cooper, Appl. Phys. Lett. 103, 241913 (2013).

${ }^{49}$ A. D. Darbal, R. D. Narayan, C. Vartuli, G. Lian, R. Graham, F. Shaapur, S. Nicolopoulos, and J. K. Weiss, Microsc. Microanal. 19, 702-703 (2013).

${ }^{50}$ M. U. Farooq, R. Villaurrutia, I. MacLaren, H. Kungl, M. J. Hoffmann, J. J. Fundenberger, and E. Bouzy, J. Microsc. 230, 445-454 (2008).

${ }^{51}$ D. Cooper, N. Bernier, and J.-L. Rouvière, Nano Lett. 15, 5289-5294 (2015).

${ }^{52}$ D. Cooper, T. Denneulin, N. Bernier, A. Béché, and J.-L. Rouvière, Micron 80, 145-165 (2016).

${ }^{53}$ S. Estradé, J. Portillo, J. Mendoza, I. Kosta, M. Serret, C. Müller, and F. Peiró, Micron 43, 910-915 (2012).

${ }^{54}$ A. D. Darbal, R. D. Narayan, C. Vartuli, T. Aoki, J. Mardinly, S. Nicolopoulos, and J. K. Weiss, Microsc. Microanal. 20, 1066-1067 (2014).

${ }^{55}$ G. Isella, D. Chrastina, B. Rössner, T. Hackbarth, H. J. Herzog, U. König, and H. von Känel, Solid-State Electron. 48, 1317-1323 (2004).

${ }^{56}$ M. M. Mirza, H. Zhou, P. Velha, X. Li, K. E. Docherty, A. Samarelli, G. Ternent, and D. J. Paul, J. Vac. Sci. Technol. B 30, 06FF02 (2012).

${ }^{57}$ R. W. Millar, K. Gallacher, A. Samarelli, J. Frigerio, D. Chrastina, G. Isella, T. Dieing, and D. J. Paul, Opt. Express 23, 18193-18202 (2015).

${ }^{58}$ M. Schaffer, B. Schaffer, and Q. Ramasse, Ultramicroscopy 114, 62-71 (2012).

${ }^{59}$ D. Tomus and H. P. Ng, Micron 44, 115-119 (2013).

${ }^{60}$ E. F. Rauch, J. Portillo, S. Nicolopoulos, D. Bultreys, S. Rouvimov, and P. Moeck, Z. Krist. Cryst. Mater. 225, 103-109 (2010).

${ }^{61}$ S. Reboh, P. Morin, M. J. Hÿtch, F. Houdellier, and A. Claverie, APL Mater. 1, 042117 (2013).

${ }^{62}$ A. Ghrib, M. El Kurdi, M. de Kersauson, M. Prost, S. Sauvage, X. Checoury, G. Beaudoin, I. Sagnes, and P. Boucaud, Appl. Phys. Lett. 102, 221112 (2013).

${ }^{63}$ M. M. J. Treacy, J. M. Gibson, and A. Howie, Philos. Mag. A 51, 389-417 (1985).

${ }^{64}$ R. K. Ham, Philos. Mag. 6, 1183-1184 (1961).

${ }^{65}$ D. M. Norfleet, D. M. Dimiduk, S. J. Polasik, M. D. Uchic, and M. J. Mills, Acta Mater. 56, 2988-3001 (2008).

${ }^{66}$ K. Iakoubovskii, K. Mitsuishi, Y. Nakayama, and K. Furuya, Microsc. Res. Tech. 71, 626-631 (2008).

${ }^{67}$ A. J. Craven, J. Bobynko, B. Sala, and I. MacLaren, Ultramicroscopy 170, 113-127 (2016).

${ }^{68}$ R. Geiger, J. Frigerio, M. J. Süess, D. Chrastina, G. Isella, R. Spolenak, J. Faist, and H. Sigg, Appl. Phys. Lett. 104, 062106 (2014).

69. Liu, J. Michel, W. Giziewicz, D. Pan, K. Wada, D. D. Cannon, S. Jongthammanurak, D. T. Danielson, L. C. Kimerling, J. Chen, F.Ö. Ilday, F. X. Kärtner, and J. Yasaitis, Appl. Phys. Lett. 87, 103501 (2005).

${ }^{70}$ A. Garner, A. Gholinia, P. Frankel, M. Gass, I. MacLaren, and M. Preuss, Acta Mater. 80, 159-171 (2014)

${ }^{71}$ M. Reisinger, J. Zalesak, R. Daniel, M. Tomberger, J. K. Weiss, A. D. Darbal, M. Petrenec, J. Zechner, I. Daumiller, W. Ecker, B. Sartory, and J. Keckes, Mater. Des. 106, 476-481 (2016).

${ }^{72}$ A. Ghrib, M. El Kurdi, M. Prost, S. Sauvage, X. Checoury, G. Beaudoin, M. Chaigneau, R. Ossikovski, I. Sagnes, and P. Boucaud, Adv. Opt. Mater. 3, 353-358 (2015). 